\title{
Wavelet Methods and Pattern Recognition for Clinical Image Fusion
}

\author{
${ }^{1}$ Arulmurugan Ramu and ${ }^{2}$ Anandakumar Haldorai \\ ${ }^{1}$ Presidency University, India. \\ ${ }^{2}$ Sri Eshwar College of Engineering, India \\ 1arulmr@gmail.com, 2anandakumar.psgtech@gmail.com
}

\author{
Article Info \\ Journal of Biomedical and Sustainable Healthcare Applications (http://anapub.co.ke/journals/jbsha/jbsha.html) \\ Doi: https://doi.org/10.53759/0088/JBSHA202101007 \\ Received 28 August 2020; Revised form 25 September 2020; Accepted 29 October 2020. \\ Available online 05 January 2021. \\ (C)2021 Published by AnaPub Publications.
}

\begin{abstract}
This research focuses of the efficacious wavelet-based methodology for clinical image fusion that is established by considering the human visual system, including the physical effects of the wavelet coefficients. Once the clinical images that have to be fused have been decomposed via the transforms of wavelet, different systems of fusion for integrating these coefficients are projected. The coefficients in the lower frequencies are chosen with the visibility-centered system, and those coefficients with the highest frequency bands are chosen using the variance-oriented approach. To effective mitigate the issue of noise and guarantee homogeneity of an image, which is being fused, coefficients are typically done based on the application of the window-centered verification process. The images are lastly structured using the inverse wavelet transforms with the composite coefficient. To effectively assess and effectively prove the effective applicability of the proposed methodology, experimentation series and comparison of the fusion approaches are done. The results of the experimentation on the real and simulated clinical images show that the projected approach is effective and is capable of yielding the proposed results of the fusion process.
\end{abstract}

Keywords - Competed Tomography (CT), Position Emission Tomography (PET), Discrete Wavelet Transform (DWT)

\section{INTRODUCTION}

Clinical imagery has become a critical aspect of a wide range of operations, involving diagnostic, analysis, and management, thanks to fast advancements in high-technology and contemporary equipment. Multimodality medical records, such as the X-ray, Position Emission Tomography (PET), Magnetic Resonance Angiography (MRA), Magnetic Resonance Imaging (MRI) and Competed Tomography (CT) pictures, are required to sustain more reliable medical data for doctors dealing with clinical diagnosis and assessment. The data provided by these multi - modality clinical pictures is generally complimentary, but it may sometimes be contradictory. The CT picture, for instance, may offer less deformation for strong materials such as bones and implants, but it cannot identify physical alterations, while the MR picture can offer healthy and pathologic soft material data, but it cannot encourage the bone details. In this situation, a single picture type may not be adequate to give doctors with precise medical needs. As a result, the merging of multidimensional clinical pictures is required, and it has emerged as a promising but difficult study topic in recent decades.

The technique of combining several input pictures and some of their attributes into a single picture without introducing deformation or losing of data is known as image synthesis. Visual fusion is the process of combining complementary and repetitive data from several pictures to produce a reconstructed image outcome [1]. As a result, the new picture produced should provide a more precise representation of the scenario than some of the personal reference photos, and is more suited for human and artificial interpretation, as well as subsequent picture computation and assessment activities. When it comes to clinical image compression, the fusion of pictures may frequently reveal new medical data that was not visible in the individual scans. Another benefit is that it may save money on storage by keeping just a single fused picture rather than many source photos.

Many approaches for picture fusion have been suggested in the research so far, and provide a comprehensive review of these approaches. Image fusion techniques may be classified into three groups based on the level at which the combining process occurs: pixel layer or sensory layer, deep features, and decision stage. The majority of image compression projects utilize pixel level-based techniques since the pictures used include the actual observed values and the procedures are relatively efficient and simple to execute. As a result, pixel-level merging is still a problem in this work, and when the words "picture merger" or "merging" are used, pixel-level combination is meant. Section II provides an analysis of the literature review regarding aspect of image fusion. The remainder of the paper is organized as follows: Section III presents a critical analysis of the paper whereby image fusion and the wavelet transforms; and the proposed methodology are presented. Lastly, Section IV concludes the paper.

\section{LITERATURE REVIEW}

In [2], researchers argue that taking the mean of the two pictures pixel by pixel is the easiest method of image compression. This technique, nevertheless, often has unfavorable negative consequences, such as decreased distinction. 
The modified mean method is a more stable solution for pixel layer merger. The arithmetic mean of the matching input image is utilized to calculate the fused pixel in this technique. Weight estimates, on the other hand, almost always require a user-defined threshold.), principal component analysis (PCA), intensity-hue-saturation and the Brovey transformation are some of the other techniques that have been established. These methods are simple to grasp and put into practice. These techniques can produce high geographical resolution-fused biomedical image; however, they potentially neglect the superior quality of the spectrum dataset that is certainly fundamental for virtual identification of biomedical image fusion.

Dahamsheh and Aksoy [3] posit that an artificial neural network (ANN) was used to perform picture fusion. The effectiveness of ANN, on the other hand, is dependent on the sample pictures, which is a disadvantage fused the pictures using a scientific technique, although the deformation is represented as a combination of Gaussian Probability Density Function (PDF) that is a restricting constraint in his technique. Since real world artifacts often include features at a variety of sizes or resolution, and multi-resolution or multi-scale methods may help utilize this feature, multi-resolution methods have sparked increased interest in picture merging.

Bhatnagar and Vyas [4] present that the Pyramids transformation and wavelet transform are two types of multiresolution methods. The input pictures are first converted into multi-resolution pyramid representations before being used in the pyramid fusion. The fusion procedure then uses a specific fusion rule to build a novel compressed pyramid from the pyramid meant for image input. An inverse multi-resolution transform is employed to rebuild the fused picture in the end The Laplacian, gradient, contrast, morphological and the ration-to-low-pass pyramid are some examples of this technique. However, since the pyramid technique does not include any typical spatial orientation selection in the process of decomposition, the aforementioned approaches typically amount to a block effect on the final results.

Arjen [5] previously employed the morphology pyramidal approach to integrate the MR and CT pictures, although this approach may sometimes result in a lot of undesired borders. Another variety of multi-resolution fused approaches is the wavelet-based technique, which often uses the Discrete Wavelet Transform (DWT) in fusing. The DWT of pixel impulses offers a non-redundant image description, which enables for enhanced spectral and spatial localization of pixel data in comparison to earlier multi-resolution models. The results show that DWT systems outperform pyramidal systems in terms of directed data, blocking distortions, and signal-to-noise ratio. As a consequence, the wavelet-oriented methodology has been utilized for biomedical image fusion and two detailed evaluations are available. Despite the fact that there exists a lot of adaptive filtering integration work that is being accomplished today, the bulk of it is centered on far images, multi-focus pictures, and optical pictures, with clinical imaging receiving less attention.

To fuse the medical images, Thakur, Ambhore and Sapkal [6] used a wavelet-oriented methodology with maximumselection union protocol that potentially is the same to the Burt method. The methodology suffers from the issues of distortion and noise as a consequence of the larger contrast. The modal maximal selection criteria for the discrete wavelet parameters were used by the researchers in the clinical picture merging. The demerit of this approach is that is considers the pixel (wave coefficient) value when deciding how to generate the merged image.

Deng, Dang and Li [7] recently developed a balanced adaptive filtering strategy for PET and CT computer vision. Their solution, on the other hand, ran into the issue of picking the value variables; in other words, their approach was dependent on the user's values. As a consequence, the merged results will vary depending on the weights employed. A new wavelet-based approach for clinical picture fuse is presented in this work, which is totally automatic. The fundamental contribution of this study is that once the wavelet decomposition has been used to decompose the original pictures, the parameters of the high and low frequency sections are computed using alternative fusion algorithms.

This innovative approach takes into consideration not just the wave parameters' physical significance but also the peculiarities of the human visual systems. As a result, the reduced and elevated zones' parameters are handled differently: the former is chosen using a visibility-based strategy, while the latter is chosen using a maximal localized variation method. Furthermore, all parameters are ultimately verified for uniformity to eliminate the existence of noise and assure the uniformity of the fused picture. An inverted wavelet decomposition using parameters from all frequency bands may then be utilized to create the merged picture. The paper makes and verifies both intuitive and statistical performance assessments. Section III below presents a critical analysis of the research.

\section{CRITICAL ANALYSIS}

Image Fusion Centred on the Wavelet Transforms

Yao and Zhang [8] came up with the idea for wavelet-based multi-resolution assessment and developed the concept behind it. A statistical technique that may discover local characteristics in a signal transmission is the waveform transformation. It may also divide two-dimensional (2D) data into several levels of resolution for multi - resolution investigation, such as 2D gray-scale picture impulses. Suggested technique, information reduction, feature recognition, and picture fusion are just a few of the applications for the wavelet based. The feature extraction image compression approach is extensively discussed and analyzed in this section.

\section{The Wavelet Transform}

Wavelet transform provide a scheme for effective decomposition of signals with every dimension fundamentally correlating to finer resolutions or lower- and relatively high bands. Discrete and continuous transformations are the two primary types of transformations. The DWT, which repeatedly corresponds to two filtering banks (with downward sampling) to the lower pass-bands, is certainly fundamental (previously the initial signals). The minimized band at the minimal resolution and the higher pass band acquired at every phase, make up the wavelet representations. This transformation is non-redundant and reversible. DWT represents a spatial-frequency form of decomposition, which presents a more flexible resolution evaluation of images. In 1D (one-dimension), the foundational basis of DWT is to 
evaluate and signify the signals as wavelet superposition. Consider that the discrete signals are signified by $f t$ the decomposition of wavelet is shown by (1):

$$
f t=\sum m, n c_{m} n^{\varphi}, n t
$$

Whereby $\varphi_{m} n^{t}=2^{(-) m} \varphi\left[2^{(-) m} t-n\right]$ and " $n$ " and " $m$ " are considered as integers. There are critical selections of $\varphi$ in the case that $\varphi_{m} n^{t}$ integrates the orthonormal aspect, in the case that the coefficient of the wavelet transform is potentially obtained by the interior mathematical expression (2):

$$
C_{n, m}=\left[f, \varphi_{n, m}\right]=\int \varphi_{n, m} t f t \quad d t
$$

To effectively establish the multi-resolution evaluation, the scaling element $\emptyset$ is required, integrating the translated and dilated version: $\emptyset_{m} n^{t}=2^{(-) m} \emptyset\left[2^{(-) m} t-n\right]$. In reference to the features of the scaling space that span by $\varphi$ and $\emptyset$, the signals $f t$ could be decomposed to the course segment and the description of different dimensions by effectively projecting it only the spaces that corresponds. In that regard, to effectively identify this decomposition, more coefficients are needed at every scale: $\partial_{n, m}$ At ever $\partial_{n, m}$ and $\partial_{n, m-1}$ illustrates the estimation of the $f$ function at $2^{m}$ and the $2^{m-1}$ resolution, in a respective manner, whereas the $C_{n, m}$ shows the data loss when shifting from a single approximation form to another. To effectively obtain the $\partial_{n, m}$ and $C_{n, m}$ at every position and scale, a function of scaling is required that is illustrated in (2). The coefficient in approximation and the wavelet coefficient can therefore be gotten.

$$
\begin{aligned}
\partial_{n, m} & =\sum_{k} h_{2 n-k^{\partial} m-1}, k \\
C_{n, m} & =\sum_{k} g_{2 n-k^{\partial} m-1}, k
\end{aligned}
$$

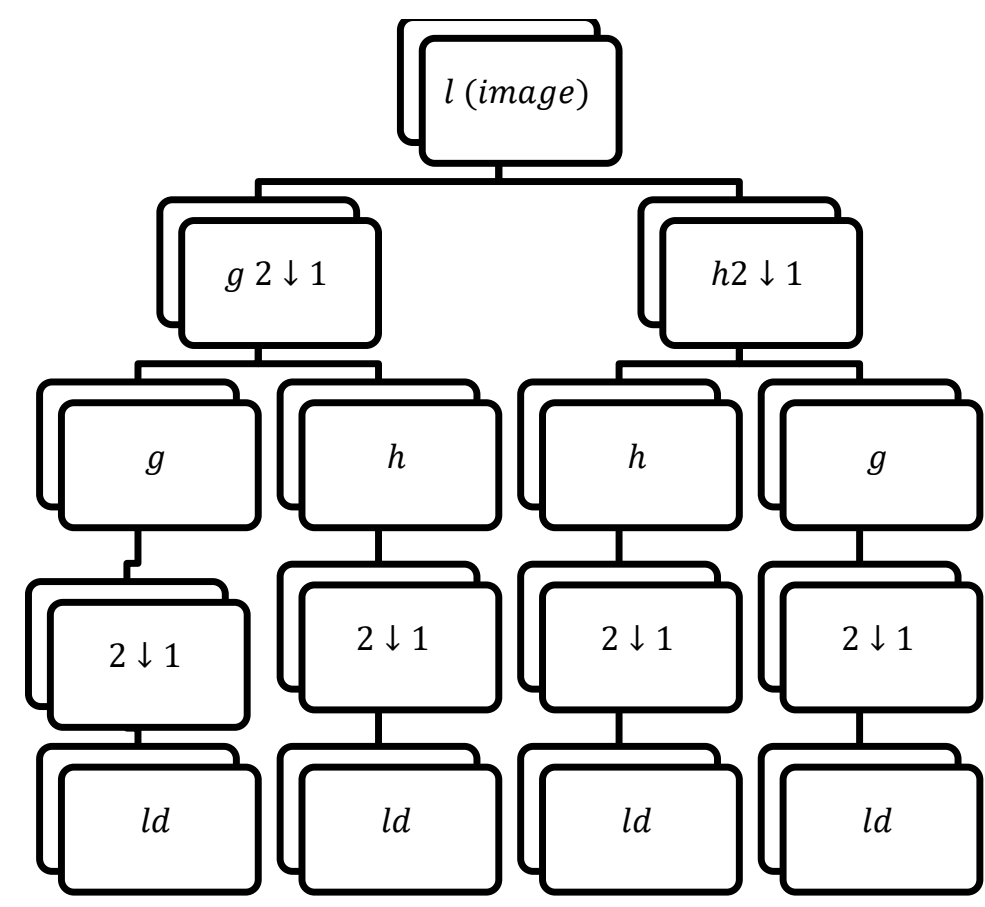

Fig 1. One phase of 2D DWT resolutional image decomposition

Whereby $h_{n}$ represents the low-pass filter (FIR) whereas $g_{n}$ represents the FIR filter that is high-pass. To restructure the initial signal, the assessment filter could be chosen from the bi-orthogonal collection that relate to the synthesis filter. The synthesis filter $\mathrm{g}$ and $\mathrm{h}$ can be utilized to effective restructure the signals based on the application of the expression (5).

$$
\partial_{m-1,} l f=\sum_{n}\left[h_{2 n-l^{\partial} n, m}, f+g_{2 n-l^{c} m, n f}\right]
$$

Expressions (4) and (3) are applied by down-sampling and filtering. Converse to that, (5) is applied by the previous filtering and up-sampling. As for the 2D DWT, the 1D DWT is done first on the row and column of the datasets by downsampling and separate filtering. This amounts to a single set of approximated coefficients $l_{\partial}$ and the three categories of the coefficient details, as indicated in Fig 1 below whereby $l_{d}, l_{c}$ and $l_{b}$ signify the diagonal, vertical and horizontal image directions respectively. As for the languages of the filter theorem, the four sub-pictures tend to correspond to the LtL (Low-to-Low) output, LtH (Low-to-High), HtL (High-to-Low) and the HtH (High-to-High) bands. Through recursively 
utilizing similar systems to LtL bands, the multi-resolution form of decomposition with the desired dimension can possibly be accomplished. In that regard, DWT in consideration to the decomposition " $\mathrm{K}$ " dimensions will incorporate $M=$ $3 \times K+1$ bands of frequency. Fig 2 indicates the 2D structure of the wavelet transforms with two distinct levels of decomposition. It is relevant to consider that for the " $\mathrm{K}$ " transforms of decomposition, there is a single band with low frequency $\left\lceil L t L^{K}\right\rceil$ in Fig 2; the remained of the bands are considered to be of high frequency in a particular level of decomposition.

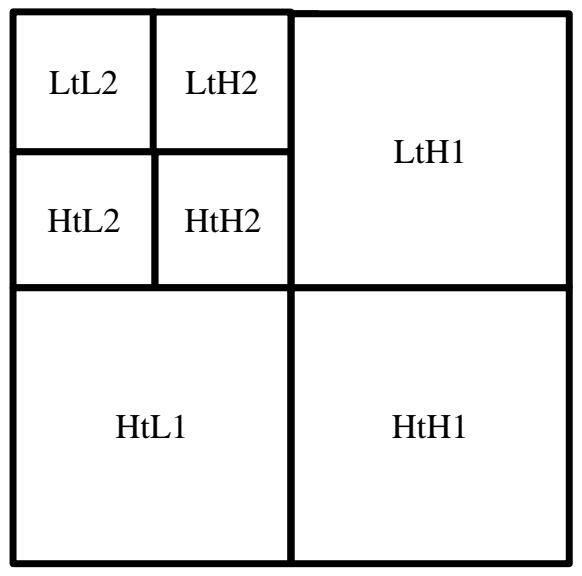

Fig 2. 2D DWT structures with labeled bands in a two-dimensional decomposition

Fusion Aspect with the Wavelet Transforms

Figure 2 is a graphic representation of the wavelet-based merging approach, which is used to better comprehend the idea and process in this part. The fundamental principle behind wavelet-based image compression is to do a multi-resolution breakdown on each reference picture, then combine the components of both the low- and high-frequency bands using an union algorithm, as shown in the center column of Fig 3. Optimal sampling strategy is the most often utilized fusion rule. The biggest objective wave parameter at each position in the input pictures is selected as the value at the site in the reconstructed images in this basic technique. The contrast enhancement is then created by conducting an inverse DWT (IDWT) on the merged wavelets. As a result, the comprehensive fusion stages centered on the wavelet transformation may be described below, as indicated in Fig 3.

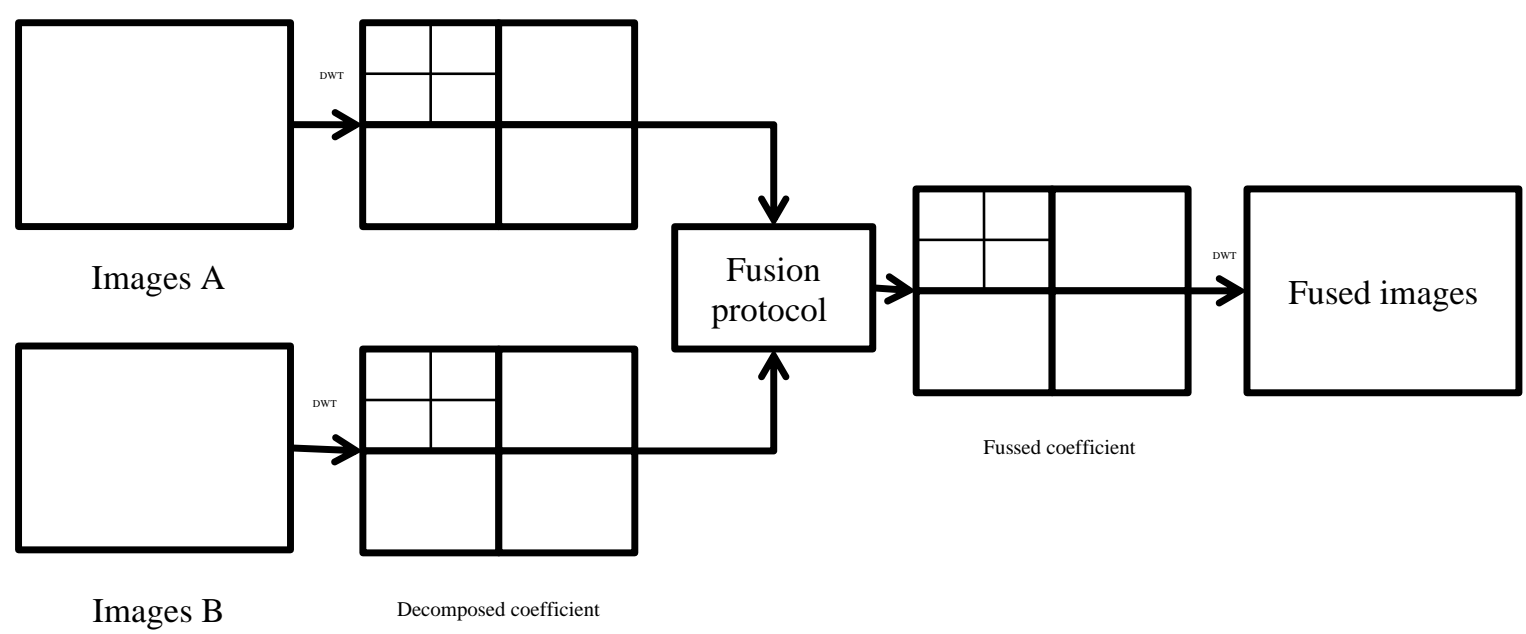

Fig 3. Comprehensive fusion stages centered on the wavelet transformation

- Phase 1: Images that need fusing have to be registered to effectively ensure pixel alignment

- Phase 2: The images are therefore decomposed to the wavelet changed images in a respective manner with respect to the wavelet transform. The changed images with the k-dimension decomposition will integrate a single portion of lower frequency (LtL band) and the $3 \mathrm{~K} \mathrm{LtH}$ bands, $\mathrm{HtL}$ bands and $\mathrm{HtH}$ bands.

- $\quad$ Phase 3: The transforms coefficient of various bands and portions are done with particular fusion protocol.

- Phase 4: The fusion picture is structured by performing wavelet transforms that are inverse on the integrated transforms of coefficients from Phase 3. 
The Proposed Fusion Methodology

The fundamental stage in image compression depending on the wave is statistic conjunction, specifically, the merging rule, as illustrated in the fuse sequence, Figure 2, since it will determine how to combine the variables in an acceptable manner so that an elevated fused picture may be created. As a result, the fusing rule construction is the most important aspect of this kind of picture fusion technique, and should be given greater emphasis. Different segmentation rules have been developed throughout the decades, which may be classified into pixel-based and window-based methods. The above mentioned maximum selection approach is a frequently used pixel-based fusion rule. This approach can extract the important information from the source photos, but it is subject to interference and artefacts since it aims for better contrast. As a consequence, certain distortion and artifacts are readily incorporated into the fused picture using this process, lowering the image quality as a result. Another pixel-based technique is the averaging fusion rule, which may help to stabilize the fusion outcome. This technique, on the other hand, is known to blur an image and even minimize the contrasts of features, which prevail in a single image.

More complicated systems that utilizes, such as window-based or geographical area, are also suggested since these systems are more resistant to picture misregistration than pixel-based methods. A window-based weighted mean fusion rule was presented by Seyedzadegan and Othman [9]. The values in this approach, nevertheless, are based on a userdefined threshold. By examining the largest relative variation value of the center variables inside a window, Li and Yang [10] employed a location-based high selection methodology to effectively identify those kinds of data inputs that are probable to integrate important data. Even though this technique has been shown to be superior to the pyramid-based approach, it has the drawback of treating the wavelet transform of both the low- and high-frequency regions in the same manner. Nevertheless, in many situations, a person is the final user or translator of the merged picture. As a result, while fusing images, human culture should be taken into account. Visual systems have distinct sensitivity to wavelet transform of low contrast band and high definition band, as per human visual systems current theories. As a result, the aforementioned fusion rules, which consider all coefficients in the same manner, will have certain drawbacks.

However, as the primary goal of this article is to effectively fuse multi-dimensional biomedical images, the features of the biomedical images must also be taken into account. An illustration of the initial CT and MR scans is shown in Fig 4. It is obvious from Fig 4 that image from the CT scale provide a clearer bone dataset but no softer tissue knowledge, while the MR scan gives detailed soft - tissue information but no fracture details. That is to state, the identical thing shows extremely clearly in both clinical photos. As a result, the approximation picture (reduced band) and the detailed picture (elevated bands) may have radically distinct physical meanings when dissected by wavelet based. Depending on this and the preceding study, this work proposes a novel clustering algorithm for computing wavelet transform that addresses the low- and high-frequency bands independently using various fusion strategies. The low band coefficients are chosen using a visibility-based method of selecting, whereas the high-frequency band coefficients are chosen using a maximal windowbased variation selection strategy. A window-oriented consistency checks are therefore applicable to the metrics taken from the various bandwidths to effectively mitigate the issue of disturbance and thereby ensure a homogenization of the images to be fused. Fig 5 illustrates the entire sequence of our proposed hybrid rule.

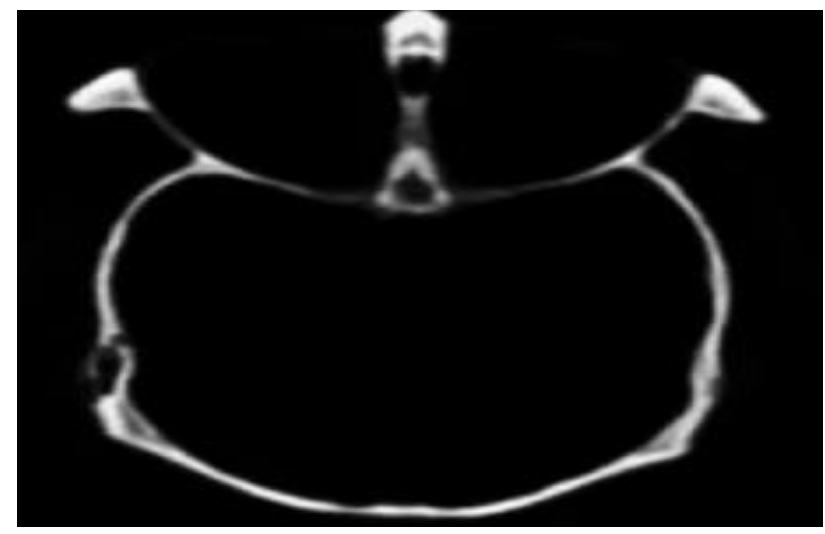

Fig 4. Initial CT medical image

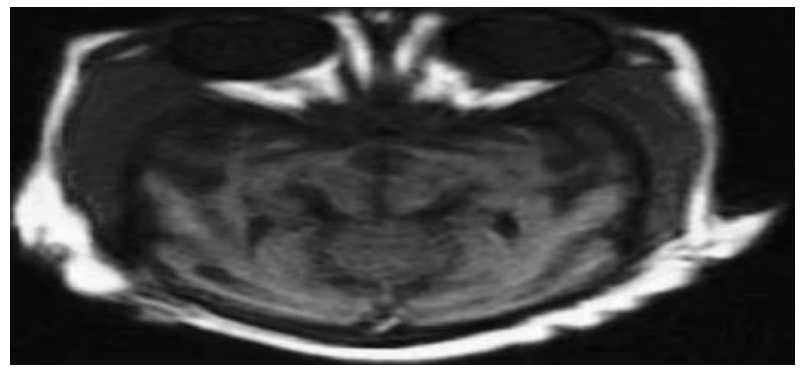

Fig 5. Initial MR medical image 


\section{Low-Frequency Bands Fusion}

In this research, to effectively abridge the definitions of several alternatives available in formulating the rules of fusion, we consider two different source biological images $(\mathrm{x}, \mathrm{y}, \mathrm{z})$ as the images to be fused. The approach could be fundamentally extended to more than two distinct pictures. In general, $l$ (images) have their distinct Multi-Scale Decompositions (MSDs) representations denoted by $D_{l}$. Therefore, we shall meet $D_{x}, D_{y}$ and $D_{z}$. Considering $p=(l, k, n, m)$ shows the indexes that correspond to the MSDs coefficients, whereby $\mathrm{n}$ and $\mathrm{m}$ shows the spatial positions in a particular frequency band denoted by $k$ signifies the decomposition levels and $l$ is the band frequency of MSDs representations.

In that regard, $D_{l} p$ repreents the valuation of MSDs of the coefficients that correspond at the $\mathrm{n}$ and $\mathrm{m}$ positions within the $\mathrm{k}$ level of decomposition and the $l$ band of frequency. Because the lower frequency band represents the initial image at a course level of resolution, it could be termed as sub-sampled and smoothed version of the initial images. In that regard, more dataset of the images' sources is effectively maintained in the low band. With respect the past analysis, in this case for the lower-frequency band, the fusion system that opts to the highest localized visibility is effectively proposed. This approach is retrieved from and is effectively stimulated by the concept that the systems of human visuals are more sensitive to the present contrasts. In that case, this approach can be probably to provide critical details to the human eye. The rule of fusion first assesses the window-based visibility of the coefficient in the low band of frequency. The visibility of the wavelet-based coefficients can be obtained as shown below:

$$
\begin{aligned}
V I p & =\frac{1}{w^{2}} \sum j, i \in B_{w} \Delta\left(D(p) . \frac{D(i+m, j+n, l, k)-D(p)}{D(p)}_{D(p)}=\frac{1}{w^{2}} \sum_{j, i} \in B_{w}\left(D(i+m, j+n, l, k), \Delta\left(D(p)=\frac{1}{D(p)}^{a}\right.\right.\right.
\end{aligned}
$$

Whereby $B_{w}$ represents the w by w block, $\Delta(D(p)$ represents the weighted factor, VI $p$ represents the block visibility, $a$ represents the visuals constant retrieved by the perceptual experimentations, and its typical range from 0.60 to 0.70 . After evaluating the visibilities of the various coefficients in the bands with lower frequency, the correspondent coefficient with high magnitude of visibilities are therefore selected into the fused pictures as provided below:

$$
D z(p)=\begin{aligned}
& D_{x} p, V I_{x} p \geq V I_{y} p \\
& D_{y} p, V I_{x} p<V I_{y} p
\end{aligned}
$$

\section{Band Fusion of Higher Frequency}

Because the goal of picture fusing is to maintain the features of input pictures such as corners, arcs, and regional borders, it is often assumed that the high-frequency bands hold the majority of the picture qualities. As a result, finding effective techniques to combine the information of input photos is critical. The traditional method of selecting high-frequency variables relies only on their exact amount, with the nearby variables being ignored. However, since each pixel in a picture must have some relationships with its neighbors, an MSD coefficient will have relationships with its neighbors as well. Furthermore, it is clear to see that the visual system attention in the high definition area is focused on the observation of variations in contrasts among areas on the corners that divide these sections, pertaining to human visual systems. As a result, an effective approach for high-frequency groups should result in large values at the edges. We suggest a strategy for selecting high-frequency components depending on the aforementioned analysis, which involves estimating the variation in a neighborhood. The following is a description of the process:

$$
\begin{gathered}
\partial_{l} p=\frac{1}{T \times S} \sum_{s=-s / 2}^{s / 2} \sum_{t=t / 2}^{t / 2} D_{l}(s+m, t+n, l, k)-\operatorname{average~} l(p)^{2} \\
\quad \text { average } l(p)=\frac{1}{T \times S} \sum_{s=-s / 2}^{s / 2} \sum_{t=t / 2}^{t / 2} D_{l}(s+m, t+n, l, k)
\end{gathered}
$$

Whereby $T \times S$ represents the closest size, while the average $l(p), \partial_{l} p$ represents the average valuation and the variance valuation of the coefficient that has been centred at " $n$ " and "w" in the windows of $T \times S$, in a respective manner. Therefore, the fusion system utilized for the high-frequency bands could be provided as shown below (11).

$$
D z(p)=\begin{aligned}
& D_{x} p, \partial_{x} p \geq \partial_{y} p \\
& D_{y} p, \partial_{x} p<\partial_{y} p
\end{aligned}
$$

It is fundamental to consider once again that the bands of higher frequency referenced herein integrate the diagonal, horizontal and vertical images of higher frequency. In that regard, the process of fusion must be done in all the three respective domains.

\section{Verification of Consistency}

As shown by the preceding subcategories, the maximal selected methods choose all of the parameters in both the minimum and maximum bands, but as we know, the maximal select methodology is influenced by noise. Moreover, since we deal with the variables independently, our approach cannot ensure that the resulting fused picture is homogeneous. As a result, a consistent verification technique is employed to guarantee that the dominating characteristics are included into the fused 
picture as thoroughly as feasible. This effort is most likely to be based on popular filtering. The variables in the composites MSD are subjected to window-based verification (WBV) [11] in this article. A tiny frame focused at the present coefficient location is used by the WBV.

WBV integrates the smaller window integrated at the current coefficients' position. The WBV rule is that in the instance that the composite MSD coefficient comes from the image " $x$ ", but a number of the closest coefficients with the windows come from the image " $y$ ", then the middle samples are transformed to come from " $y$ ". In application, the rule is applied in the binary decisions map and thus succeeded by the application of most filters. The fusion coefficient is lastly retrieved by the novel binary decision maps. The whole procedure can be provided as shown below (12).

$$
\begin{aligned}
& D_{x} p=\underset{w \in w}{\operatorname{maximum}\left[D_{x}(w, p)\right],} \\
& D_{y} p=\begin{array}{c}
w \in W \\
\operatorname{maximum}\left[D_{y}(w, p)\right], \\
w \in w
\end{array}, \\
& \partial_{x} p=1\left[\begin{array}{c}
w \in W \\
\left.D_{x}(p)>D_{y} p\right]
\end{array},\right.
\end{aligned}
$$

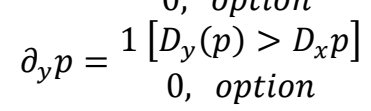

$$
\begin{aligned}
& \partial_{\frac{*}{x}} p=\begin{array}{c}
\sum\left[\partial_{x}(p) \geq 5\right] \\
0, \text { option }
\end{array}, \\
& \partial_{\bar{y}}^{*} p=1-\partial_{\frac{x}{*}} p, \\
& \stackrel{y}{D_{z}} p=\partial_{\frac{*}{x}} p D_{x}^{x} p+\partial_{\frac{*}{y}} p D_{y} p
\end{aligned}
$$

Whereby "w" is a 3 by 3 window, while the valuation of the most of the filters is reset to 5

Using the process shown above, the integrated coefficient is therefore considered by the inverted wavelet transforms, while the fused images can be obtained in a consequent manner. Therefore, the phases of this fusion methodology can be summarized as shown below [12-17].

- $\quad$ Phase 1: Registration of the multi-modal clinical images

- Phase 2: Decomposition of the images to the three and four wavelet plans (levels of resolution)

- Phase 3: Wavelet coefficient of the lower frequencies are selected by the $8^{\text {th }}$ and $9^{\text {th }}$ equations and the wavelet coefficients of the high-band frequency is selected by the $9^{\text {th }}$ equation.

- Phase 4: The coefficients of the high and low frequencies are fulfilled by the consistencies implementation of equation 11.

- $\quad$ Phase 5: Performance of the inverse wavelet transforms with the integrated coefficient obtained in Phase 4.

$\bullet$

\section{CONCLUSION}

In conclusion, the fusing of the multi-modal clinical images is vital when it comes to its applications in the medical setting. This approach is utilized in supporting the production of more precise data compared to individual source images. This research presents a wavelet-centered methodology for the clinical image fusion that integrates three fundamental phases. In the $1^{\text {st }}$ phase, the biomedical images, which have been compressed, are effectively decomposed into sub-images through the process of wavelet transformation. In the second phase, one the features of the human visual systems have been considered and the physical effect of the wavelet-based coefficient observed, the coefficient of the lower frequency bands and the higher frequency band are done considering the various fusion approaches: the previous one is chosen based on the application of the maximum visibility system, and the latter is chosen based on the maximum localized variance protocol. To enhance the quality of the obtained images, the integrated coefficient is therefore considered by the window-centered consistency verifications. In the final phase, the fused images are established using the inverse wavelet-based transform with the fused coefficient. The undertaking of the projected methodology is both quantitatively and qualitatively compared with prevailing methodologies of fusion.

\section{References}

[1]. D. Sannen and H. Van Brussel, "A multilevel information fusion approach for visual quality inspection", Information Fusion, vol. 13, no. 1, pp. 48-59, 2012. Doi: 10.1016/j.inffus.2011.02.005.

[2]. "High Capacity Image Steganography using Pixel Value Differencing Method with Data Compression using Neural Network", International Journal of Innovative Technology and Exploring Engineering, vol. 8, no. 12, pp. 1800-1804, 2019. Doi: 10.35940/ijitee.12839.1081219.

[3]. A. Dahamsheh and H. Aksoy, "Artificial neural network models for forecasting intermittent monthly precipitation in arid regions", Meteorological Applications, vol. 16, no. 3, pp. 325-337, 2009. Doi: 10.1002/met.127.

[4]. R. Bhatnagar and A. Vyas, "COMPARATIVE STUDY OF WAVELET TRANSFORM AND WAVELET PACKET TRANSFORMATION IN IMAGE DENOISING USING THRESHOLDING TECHNIQUE", International Journal of Technical Research \& Science, vol. 6, no. 1, pp. 10-15, 2021. Doi: 10.30780/ijtrs.v06.i01.002.

[5]. V. Arjen, "Influence of neuronal morphology on pyramidal cell burst firing", Frontiers in Neuroinformatics, vol. 2, 2008. Doi: 10.3389/conf.neuro.11.2008.01.019.

[6]. K. Thakur, P. Ambhore and A. Sapkal, "Novel Technique for Performance Improvement of the Wavelet based Denoising Algorithms using Rotated Wavelet Filters", Procedia Computer Science, vol. 79, pp. 499-508, 2016. Doi: 10.1016/j.procs.2016.03.064. 
[7]. Z. Deng, J. Dang and T. Li, "An Adaptive Fir Filtering Based on Balanced Realization", Advanced Materials Research, vol. 753-755, pp. 2566-2572, 2013. Doi: 10.4028/www.scientific.net/amr.753-755.2566.

[8]. C. Yao and G. Zhang, "A Multi-Resolution PID Controller Based on the Wavelet Transform", Advanced Materials Research, vol. 472-475, pp. 632-636, 2012. Doi: 10.4028/www.scientific.net/amr.472-475.632.

[9]. M. Seyedzadegan and M. Othman, "Weighted Window and Class-Based Weighted Window Methods for Per-Station TCP Fairness in IEEE 802.11 WLANs", EURASIP Journal on Wireless Communications and Networking, vol. 2010, no. 1, 2010. Doi: 10.1155/2010/593497.

[10]. Y. Li and A. Yang, "A Bayesian Criterion-based Statistic for Model Selection of Structural Equation Models with Ordered Categorical data", International Journal of Modeling and Optimization, pp. 151-157, 2011. Doi: 10.7763/ijmo.2011.v1.27.

[11]. X. Zhong and I. Ridley, "Verification of behavioural models of window opening: The accuracy of window-use pattern, indoor temperature and indoor PM2.5 concentration prediction", Building Simulation, vol. 13, no. 3, pp. 527-542, 2020. Doi: 10.1007/s12273-020-0615-4.

[12]. A. Haldorai and A. Ramu, "Security and channel noise management in cognitive radio networks," Computers \& Electrical Engineering, vol. 87, p. 106784, Oct. 2020. doi:10.1016/j.compeleceng.2020.106784

[13]. A. Haldorai and A. Ramu, "Canonical Correlation Analysis Based Hyper Basis Feedforward Neural Network Classification for Urban Sustainability," Neural Processing Letters, Aug. 2020. doi:10.1007/s11063-020-10327-3

[14]. D. Devikanniga, A. Ramu, and A. Haldorai, "Efficient Diagnosis of Liver Disease using Support Vector Machine Optimized with Crows Search Algorithm," EAI Endorsed Transactions on Energy Web, p. 164177, Jul. 2018. doi:10.4108/eai.13-7-2018.164177

[15]. H. Anandakumar and K. Umamaheswari, "Supervised machine learning techniques in cognitive radio networks during cooperative spectrum handovers," Cluster Computing, vol. 20, no. 2, pp. 1505-1515, Mar. 2017.

[16]. H. Anandakumar and K. Umamaheswari, "A bio-inspired swarm intelligence technique for social aware cognitive radio handovers," Computers \& Electrical Engineering, vol. 71, pp. 925-937, Oct. 2018. doi:10.1016/j.compeleceng.2017.09.016

[17]. R. Arulmurugan and H. Anandakumar, "Early Detection of Lung Cancer Using Wavelet Feature Descriptor and Feed Forward Back Propagation Neural Networks Classifier," Lecture Notes in Computational Vision and Biomechanics, pp. 103-110, 2018. doi:10.1007/978-3 319-71767-8_9 